\title{
Paramagnetic lonic Liquids for Measurements of Density Using Magnetic Levitation
}

\section{Citation}

Bwambok, David K., Martin M. Thuo, Manza B.J. Atkinson, Katherine A. Mirica, Nathan D. Shapiro, and George M. Whitesides. 2013. "Paramagnetic lonic Liquids for Measurements of Density Using Magnetic Levitation.” Analytical Chemistry 85(17): 8442-8447.

\section{Published Version}

doi:10.1021/ac401899u

\section{Permanent link}

http://nrs.harvard.edu/urn-3:HUL.InstRepos:12361277

\section{Terms of Use}

This article was downloaded from Harvard University's DASH repository, and is made available under the terms and conditions applicable to Open Access Policy Articles, as set forth at http:// nrs.harvard.edu/urn-3:HUL.InstRepos:dash.current.terms-of-use\#OAP

\section{Share Your Story}

The Harvard community has made this article openly available.

Please share how this access benefits you. Submit a story.

Accessibility 


\section{Paramagnetic Ionic Liquids for Measurements of Density Using Magnetic Levitation}

David K. Bwambok ${ }^{1}$, Martin M. Thuo ${ }^{1}$, Manza B.J. Atkinson ${ }^{1}$, Katherine A. Mirica ${ }^{1}$, Nathan D. Shapiro $^{1}$ and George M. Whitesides ${ }^{1,2,3 *}$

${ }^{1}$ Department of Chemistry and Chemical Biology, Harvard University,

12 Oxford St., Cambridge, MA 02138

${ }^{2}$ Wyss Institute for Biologically Inspired Engineering, Harvard University,

60 Oxford St., Cambridge, MA 02138

${ }^{3}$ Kavli Institute for Bionano Science \& Technology, Harvard University, 29 Oxford Street Cambridge, MA 02138

* Corresponding author: George M. Whitesides (gwhitesides@gmwgroup.harvard.edu) 


\begin{abstract}
Paramagnetic ionic liquids (PILs) provide new capabilities to measurements of density using magnetic levitation (MagLev). In a typical measurement, a diamagnetic object of unknown density is placed in a container containing a PIL. The container is placed between two magnets (typically $\mathrm{NdFeB}$, oriented with like poles facing). The density of the diamagnetic object can be determined by measuring its position in the magnetic field along the vertical axis (levitation height, h), either as an absolute value, or relative to internal standards of known density. For density measurements by MagLev, PILs have three advantages over solutions of paramagnetic salts in aqueous or organic solutions: (i) negligible vapor pressures; (ii) low melting points; (iii) high thermal stabilities. In addition, the densities, magnetic susceptibilities, glass transition temperatures, thermal decomposition temperatures, viscosities, and hydrophobicities of PILs can be tuned over broad ranges by choosing the cation-anion pair. The low melting points and high thermal stabilities of PILs provide large liquidus windows for density measurements. This paper demonstrates applications and advantages of PILs in density-based analyses using MagLev.
\end{abstract}

Keywords: Ionic liquid; paramagnetic ionic liquid; magnetic levitation; density; magnetic susceptibility; temperature; analytical chemistry. 


\section{INTRODUCTION}

Magnetic levitation (MagLev) is a useful new method for measuring the density of a diamagnetic object. ${ }^{1-9}$ MagLev, as developed in our laboratory, relies on paramagnetic solutions (typically generated by dissolving a paramagnetic salt — alone, or as a chelate — in water or an organic solvent), placed in a magnetic field gradient, to suspend a diamagnetic object against gravity. ${ }^{1}$ This procedure has broad generality, but also six limitations: (i) Evaporation of the solvent from a solution of paramagnetic salt will increase the concentration of the paramagnetic species, and thus the magnetic susceptibility of the solution. This evaporation of solvent complicates the use and storage of paramagnetic solutions, and in some applications requires calibration or the use of internal standards. (ii) Aqueous solutions of paramagnetic salts cannot be used for density-based measurement of water-miscible or water-soluble analytes. (iii) Paramagnetic salts have high but ultimately finite solubility in water (the limiting solubility in of $\mathrm{MnCl}_{2}$ is $\sim 4.5 \mathrm{M}$ ), and even more limited solubility in organic solvents). Water has limited ability to dissolve the paramagnetic salts at subzero temperatures $\left(<0{ }^{\circ} \mathrm{C}\right)$. These limits to solubility constrain the range of densities that can be measured using these solutions. (iv) Some organic solvents used for water-miscible analytes in MagLev (especially $\mathrm{CBr}_{4}$ for high-density samples) are toxic and/or flammable. (v) To allow for measurement of water-soluble analytes, the paramagnetic metal ion must be converted into an organic-soluble chelate to give useful solubility; this transformation requires additional synthesis steps. (vi) Measurement of very low $\left(\rho<1.00 \mathrm{~g} / \mathrm{cm}^{3}\right)$ and high $\left(\rho>3.00 \mathrm{~g} / \mathrm{cm}^{3}\right)$ densities is complicated or difficult using simple techniques (although possible with newer versions of MagLev).

Measurement of density by MagLev can be applied to objects with a wide range of sizes, shapes and physical properties. Since it requires no electricity, MagLev may be particularly 
useful in resource-limited or field settings, where analytical instrumentation should, ideally, be small, lightweight, independent of electrical power, and easy to use. ${ }^{1}$

We have demonstrated the use of MagLev for (inter alia): (i) analyses of food and water ${ }^{2}$, (ii) measurements of protein-ligand binding ${ }^{3}$, and protein-ligand dissociation ${ }^{4}$, (iii) threedimensional self-assembly ${ }^{5,6}$, (iv) density-based separations in binding events ${ }^{7}$, (v) forensics ${ }^{8}$, (vi) distinguishing chemical composition of polymers; (vii) and monitoring the course of chemical reactions. ${ }^{9}$

A measurement of density by MagLev requires three components: (i) magnet(s) configured to create both magnetic field and magnetic field gradient; (ii) a paramagnetic medium, and, (iii) one or more diamagnetic analytes. The combination of magnetic field, B, and gradient in the magnetic field (B.VB) provides the magnetic force that suspends the diamagnetic analyte against gravity. ${ }^{1}$ The paramagnetic medium has two roles: (i) its magnetic susceptibility (i.e., the extent of magnetization in response to the external magnetic field) controls the magnitude of the magnetic (levitation) force, (ii) in accordance with Archimedes' principle, its density determines the gravitational buoyancy of the diamagnetic analyte. In order to measure density accurately, the paramagnetic medium and the diamagnetic analyte must, of course, be chemically compatible and immiscible.

Replacing solutions of paramagnetic salts with paramagnetic ionic liquids (PILs) circumvents some of the limitations imposed by water (or organic fluids), often used as solvents in MagLev. The use of PILs for MagLev offers at least seven advantages: (i) Since these ionic liquids are inherently paramagnetic, they can be used without dilution; PILs thus offer higher magnetic susceptibilities than solutions of paramagnetic salts; they also eliminate errors or uncertainties due to variations in concentration of a paramagnetic solute. (ii) PILs have very low vapor 
pressures, and do not evaporate at room temperature and atmospheric pressure. (iii) The density and magnetic susceptibility of PILs are defined by their chemical components, and by the stoichiometries of those components. Since the stoichiometry is fixed, and since the PILs can be used in their pure liquid state, the magnetic susceptibility and density of each system is fixed. These two properties make it easy to recycle PILs and enable their long-term use as the levitating medium in MagLev. (iv) The chemical and physical properties of PILs can be tuned by changing the anion-cation pair (or in some cases by post-synthetic modification of the structure of one of the ions). This flexibility allows for the production of ionic liquids that are either hydrophilic or hydrophobic, low or high in melting point, viscous or non-viscous, or more or less dense than water. (v) PILs are stable over a much larger range of temperatures than most commonly used solvents. For example, 1-butyl-3-methylimidazolium tetrachloroferrate ([BMIM] $\left.\left[\mathrm{FeCl}_{4}\right]\right)$ is stable to heating up to $280^{\circ} \mathrm{C}$, and is liquid (although very viscous) when cooled as low as -88 ${ }^{\circ} \mathrm{C} .{ }^{10}$ The high thermal stabilities and large liquidus windows of PILs allows their use at high and low temperatures. (vi) PILs, like conventional ionic liquids, are expected to be generally nontoxic, non-flammable, and recyclable. ${ }^{11,12,13}$ (vii) Some PILs can be used for measurement of densities outside ranges accessible with aqueous paramagnetic salt solutions without co-solute additives (e.g., $<1.0 \mathrm{~g} \cdot \mathrm{cm}^{3},>1.5 \mathrm{~g} \cdot \mathrm{cm}^{3}$ ). (vii) PILs are readily synthesized from commercially available materials in a single step and with no need for complicated purification procedures. PILs are obtained conveniently by ion exchange, and the experimental procedures used can be adopted to achieve custom liquids that are transparent (to allow observation in MagLev experiments), and are readily scalable where large quantities are needed.

We note that PILs also have some disadvantages: (i) They are relatively new materials, and many (including all used in this paper) are not commercially available; they are also more 
expensive than paramagnetic salts (if recycled, the cost can probably fall below that of salt solutions that are not recycled). (ii) PILs have fixed compositions, and adjusting their magnetic susceptibilities and densities requires adding other species (miscible ionic liquids, salts or altering the cation-anion pair). (iii) Some PILs are quite viscous; a high viscosity increases the time it takes an object to reach its equilibrium levitation height. For instance, it takes $\sim 1$ min for a sphere with a diameter of $4.8 \mathrm{~mm}$ to reach its equilibrium height when levitating in [BMIM] $\left[\mathrm{FeCl}_{4}\right]$ paramagnetic liquid with viscosity of $18 \mathrm{cP}$. In contrast, the sphere reaches its equilibrium height in $\sim 10$ seconds when levitated in the less viscous solution of $3 \mathrm{M}$ aqueous manganese chloride.

Syntheses of several PILs have been reported ${ }^{14,15,16}$, but they have not been used with MagLev to measure density. To our knowledge, there is only one relevant report: that of the influence of a magnetic field on nitrogen bubbles moving through a paramagnetic ionic liquid. ${ }^{17}$ We anticipate that PILs, like conventional ionic liquids that are readily recycled in applications such as organic synthesis, can also be recycled in MagLev. Certain contaminants that may be absorbed during analysis can be extracted from the PILs using an organic solvent, water ${ }^{18,19}$ or supercritical fluid (e.g., carbon dioxide). ${ }^{20}$

This paper demonstrates the use of PILs as non-volatile levitation media in MagLev. We describe the synthesis of PILs (three previously reported, as well as thirteen previously unreported), by reaction of organic halides and paramagnetic metal halides as detailed previously. ${ }^{15,16}$ The anions of the PILs we have synthesized are based on iron(III), gadolinium(III), manganese(II), holmium(III), and dysprosium(III) halides. The cations are based on imidazolium, amino acid ester, and tetra-alkylammonium salts. We demonstrate that it 
is possible to adjust the magnetic susceptibility and density of the PILs by choosing the cation/anion pair and varying the temperature.

\section{RESULTS AND DISCUSSION}

Synthesis and characterization of PILs. We synthesized the PILs in nearly quantitative $(100 \%)$ yield by combining imidazolium, amino acid ester, or ammonium halides with paramagnetic metal halides (Scheme 1). These reactions can be performed with or without solvent, followed by removal of the solvent by rotary evaporation (see Supporting Information for experimental details). We characterized these PILs using elemental analysis (see Supporting Information). The PILs respond to and can be easily manipulated by an inexpensive $\mathrm{NdFeB}$ (B $0.4 \mathrm{~T}$ at the surface) magnet (Figure $\mathrm{S} 1$ ).

Thermal properties of PILs. We determined the temperature range over which PILs are liquids (i.e., the liquidus window), by measuring the glass transition $\left(\mathrm{T}_{\mathrm{g}}\right)$, and decomposition $\left(\mathrm{T}_{\mathrm{d}}\right)$ temperature of each PIL; Table 1 describes these values. All these PILs are liquids at room temperature. We measured the glass transition temperatures using differential scanning calorimetry (DSC), by monitoring the transitions of the PILs in the temperature range from $25^{\circ} \mathrm{C}$ to $-75^{\circ} \mathrm{C}$ (the detection limit of our equipment). In general, these PILs displayed a broader range

of $T_{g}$ values compared to conventional ionic liquids with narrow range of $T_{g}$ values. ${ }^{21,22}$ Seven of the PILs that we synthesized show glass transitions between $-24^{\circ} \mathrm{C}$ to $-75^{\circ} \mathrm{C}$; seven remain liquid with no observable glass transitions from room temperature to $-75^{\circ} \mathrm{C}$. The glass transition temperature for L-alanine methyl ester dysprosium hexachloride, $[\mathrm{AlaC} 1]_{3}\left[\mathrm{DyCl}_{6}\right]$, for example, is $-56^{\circ} \mathrm{C}$, with no observable melting transition; 1- butyl-2,3- dimethylimidazolium dysprosium hexachloride, $[\mathrm{BDMIM}]_{3}\left[\mathrm{DyCl}_{6}\right]$, shows neither glass transition nor melting point from room 
temperature to $-75^{\circ} \mathrm{C}$. PILs can, therefore, be used for density measurements at temperatures where aqueous paramagnetic solutions or many organic solvents would freeze (e.g., below $0{ }^{\circ} \mathrm{C}$ ). In addition, PILs (and most paramagnetic materials) have higher magnetic susceptibilities at lower temperatures ${ }^{15}$ than at room temperature, and this increase would enable measurements of density for materials with densities higher than could be measured at room temperature using the same medium.

We measured the thermal stability of each PIL at high temperatures using thermal gravimetric analysis (TGA) by monitoring the change in weight as the sample is heated from room temperature to $700^{\circ} \mathrm{C}$. The stability of PILs to decomposition upon heating depends on the cation/anion pair. For example, imidazolium-based PILs with dysprosium anions are more stable $\left(\mathrm{T}_{\mathrm{d}}, 330^{\circ} \mathrm{C}\right)$ than alanine-based PILs having similar dysprosium anions $\left(\mathrm{T}_{\mathrm{d}}, 160^{\circ} \mathrm{C}\right)$. In general, the high thermal stabilities of PILs enables their use for MagLev at elevated temperatures (e.g. above $100^{\circ} \mathrm{C}$ ), where water and most organic solvents would boil. PILs, therefore, provide a large liquidus window, $[\mathrm{BDMIM}]_{3}\left[\mathrm{DyCl}_{6}\right]$, for example, is liquid with tractable viscosity and acceptable stability for MagLev at temperatures from $-75^{\circ} \mathrm{C}$ to $330^{\circ} \mathrm{C}$.

Density-based measurements using PILs in MagLev. We measured the density of diamagnetic objects by suspending them in a container filled with a PIL, and placing this container between two $\mathrm{NdFeB}$ magnets $(5 \times 5 \times 2.5 \mathrm{~cm})$ oriented with like poles facing each other, $4.5 \mathrm{~cm}$ apart (Figure 1). ${ }^{1}$ The theory describing the levitation of diamagnetic objects due to balance of gravitational and magnetic forces is detailed elsewhere. ${ }^{1}$ In the configuration of the magnets shown in Figure 1, the vertical equilibrium position—levitation height, $h(\mathrm{~m})$ —of the diamagnetic object within the device correlates with its density $\rho_{s}$ (equation 1). 


$$
h=\frac{\left(\rho_{s}-\rho_{P I L}\right) g \mu_{0} d^{2}}{\left(\chi_{s}-\chi_{P I L}\right) 4 B_{0}{ }^{2}}+\frac{d}{2}
$$

In this equation, $\rho_{s}\left(\mathrm{~kg} / \mathrm{m}^{3}\right)$ and $\rho_{P L}\left(\mathrm{~kg} / \mathrm{m}^{3}\right)$ are the densities of the analyte and the PIL respectively, $\chi_{s}$ and $\chi_{P I L}$ (both unitless) are the magnetic susceptibilities of the sample and the PIL, respectively, $d(\mathrm{~m})$ is the distance between the magnets, $B_{0}($ Tesla, T) is the magnitude of the magnetic field at the surface of the magnets, $g\left(9.8 \mathrm{~m} / \mathrm{s}^{2}\right)$ is the acceleration due to gravity, and $\mu_{0}\left(1.26 \times 10^{-6}\right.$ T.m. $\left.\mathrm{A}^{-1}\right)$ is the magnetic permeability of free space. We used Eq. 1 , and calibrated density-standards, to determine the density $\left(\rho_{P I L}\right)$ and the magnetic susceptibility $\left(\chi_{P I L}\right)$ of each PIL (see supporting information). ${ }^{1}$ We show an example of density-standard beads levitating in 1-butyl-2,3-dimethyl imidazolium dysprosium hexachloride, [BDMIM $]_{3}\left[\mathrm{DyCl}_{6}\right]$, PIL (Figure 1).

We demonstrate that PILs can be used for accurate measurement of density for prolonged time in an open vessel (or after heating to higher temperatures followed by cooling) because they do not evaporate and their magnetic susceptibility remains constant (Figure 2). In contrast, aqueous or organic solvents evaporate over time in an open vessel (or when heated to higher temperatures) and as a result the concentration of the paramagnetic species, and magnetic susceptibility of the paramagnetic medium, changes (Figure 2).

Each PIL has its own characteristic density and magnetic susceptibility (Figure 3, see Table 1 for values). We investigated the accessible density range of each PIL by levitating beads having known density in the PILs and measuring levitation height (Figure 3). Varying the structure of the cation or anion has a predictable effect on density and magnetic susceptibility. Introduction of an additional methyl group in the imidazolium cation, for example, lowers the magnetic susceptibility and density of the PIL (Figure 3). Variation of the metal halide anion also 
changes both the magnetic susceptibility and density of the PIL; for example, [Aliq $]_{3}\left[\mathrm{HoCl}_{6}\right]$ has a slightly higher magnetic susceptibility, but significantly lower density, than [Aliq $]_{3}\left[\mathrm{HoBr}_{6}\right]$ (Figure 3, Table 1). Each PIL thus provides a levitation medium with characteristic magnetic susceptibility and density. In addition, the PILs have different polarities, and one can, therefore, choose a hydrophilic or hydrophobic PIL depending on what is required for the application at hand. We determined the aqueous solubility of each PIL qualitatively by mixing each PIL (5-10 $\mu \mathrm{L})$ with water $(1 \mathrm{~mL})$. Among the PILs we synthesized, those based on Aliquat ${ }^{\circledR}$ are hydrophobic, and those based on imidazolium and alaninium ions are hydrophilic.

Some PILs expand the range of densities that can be measured using MagLev. For instance, $[\mathrm{BMIM}]_{3}\left[\mathrm{DyCl}_{6}\right]$, has a magnetic susceptibility $\left(\chi=12.4 \times 10^{-4}\right)$; that is, about three times greater than that of saturated aqueous $\mathrm{MnCl}_{2}$ solution $\left(\sim 4.5 \mathrm{M}, \chi=4.2 \times 10^{-4}\right)$. This PIL, therefore, enables the levitation of analytes across a wider range of densities $\left(0.80<\rho<2.00 \mathrm{~g} / \mathrm{cm}^{3}\right)$ than that accessible $\left(1.20<\rho<1.50 \mathrm{~g} / \mathrm{cm}^{3}\right)$ at the highest concentration of aqueous $\mathrm{MnCl}_{2}$ solution ( 4.5 M) having no additives. We have extrapolated the linear plots of levitation height $(0-45$ $\mathrm{mm}$ ) against density, and the density range that can be measured practically may be smaller. We note that some of the PILs are quite viscous (e.g., $\eta=18 \mathrm{cP}$ for $[\mathrm{BMIM}]\left[\mathrm{FeCl}_{4}\right] \mathrm{PIL}$ ). As a result, $\mathrm{mm}$-size diamagnetic objects require a longer time ( $\sim 60$ seconds) to reach their equilibrium levitation height after being placed in the MagLev device than they do in relatively low-viscosity paramagnetic aqueous solutions ( $\sim 10$ seconds).

Effect of temperature on the magnetic susceptibility and density of PILs. The density of PILs decreases with increasing temperature. For example, the density of $\left[\mathrm{AlaC}_{1}\right]_{3}\left[\mathrm{DyCl}_{6}\right]$ decreased on increasing the temperature from $296 \mathrm{~K}$ to $323 \mathrm{~K}$ (Figure 4). This trend was expected, and is consistent with reports on temperature dependence of density for ionic 
liquids. ${ }^{23,24}$ The magnetic susceptibility of paramagnetic ions increases with decreasing temperature according to the equation: $\chi=\mathrm{C} /\left(\mathrm{T}-\mathrm{T}_{\mathrm{c}}\right)$ where $\chi$ is the magnetic susceptibility, $\mathrm{T}$ is the temperature $(\mathrm{K}), \mathrm{C}$ is the Curie constant and $\mathrm{T}_{\mathrm{c}}$ is the Curie-Weiss temperature $(\mathrm{K}) .{ }^{10}$

We illustrate, for example, that lowering the temperature of $[\mathrm{Aliq}]_{3}\left[\mathrm{HoBr}_{6}\right] \mathrm{PIL}$ to $-20{ }^{\circ} \mathrm{C}$ increases its magnetic susceptibility $\left(\chi_{\mathrm{r} . \mathrm{t} .}=6.30 \times 10^{-4} ; \chi_{-20^{\circ} \mathrm{C}}=6.95 \times 10^{-4}\right)$; this increase enables the levitation of denser beads (e.g., $1.25 \mathrm{~g} / \mathrm{cm}^{3}$ ) than could be levitated using the same PIL at room temperature (Figure S2). The temperature-dependent magnetic susceptibility and density of PILs broadens the range of densities that can be measured using the same paramagnetic medium (Figure S2). The density of the $[\mathrm{Aliq}]_{3}\left[\mathrm{HoBr}_{6}\right]$ PIL increased $\left(\rho_{\text {r.t. }}=1.10 \mathrm{~g} / \mathrm{cm}^{3} ; \rho_{-20^{\circ} \mathrm{C}}=1.15\right.$ $\mathrm{g} / \mathrm{cm}^{3}$ ); this increase reduce the need to use additives (e.g., diamagnetic co-solutes, diamagnetic ionic liquids), to adjust the density of the medium.

Correlating density and composition of isotopes and milk using PILs in MagLev. To demonstrate the applications of PILs with the use of MagLev, we show that PILs can i) correlate the density of known mixture of isotopes to isotopic composition (e.g., differences of five percent in composition can be measured for $\mathrm{D}_{2} \mathrm{O} / \mathrm{H}_{2} \mathrm{O}$ mixture) as a method to monitor isotope enrichment in mixtures (Figure 5), ii) measure differences in density between whole milk and adulterated (e.g., with melamine or water) whole milk (Figure S3, Supporting Information).

\section{CONCLUSIONS}

PILs are useful as non-volatile media for density-based measurements, using MagLev, across a broad range temperatures and densities. Some PILs have lower densities $\left(<1.00 \mathrm{~g} / \mathrm{cm}^{3}\right)$ than water; these low values enable measurement of density in a range that otherwise requires volatile organic solvents (e.g., ethanol). PILs have large liquidus window and can be used for density measurements in MagLev at low temperatures (e.g., below $0{ }^{\circ} \mathrm{C}$ ) where the aqueous 
paramagnetic solutions would be frozen or at higher temperatures (e.g., above $100{ }^{\circ} \mathrm{C}$ ) where the aqueous solvent would evaporate or boil. The magnetic susceptibility of PILs increases with decreasing temperature and allows the measurement of higher densities than could be achieved using the same PIL at room temperature.

PILs extend the use of MagLev to measure the density of diamagnetic objects. We extend the use of PILs for density-based measurement of analytes that are incompatible with aqueous or organic media (e.g., to measure composition of water isotopes, and milk products). MagLev provides a power-free method for density-based measurement of a mixture of isotopes and for screening the quality of milk in resource-limited settings. The ability to measure density differences at low temperatures can be useful for monitoring crystallization in cooled liquids.

\section{ACKNOWLEDGEMENTS}

This work was supported by the Bill \& Melinda Gates Foundation (no. 51308). We thank the Materials Research and Engineering Center (MRSEC, Harvard University contract \#: NSF-DMR 0820484) for DSC and TGA instrumentation.

\section{REFERENCES}

(1) Mirica, K. A.; Shevkoplyas, S. S.; Phillips, S. T.; Gupta, M.; Whitesides, G. M. J. Am. Chem. Soc. 2009, 131, 10049-10058.

(2) Mirica, K. A.; Phillips, S. T.; Mace, C. R.; Whitesides, G. M. J. Agric. Food Chem. 2010, 58, 6565-6569.

(3) Shapiro, N. D.; Mirica, K. A.; Soh, S.; Phillips, S. T.; Taran, O.; Mace, C. R.; Shevkoplyas, S. S.; Whitesides, G. M. J. Am. Chem. Soc. 2012, 134, 5637-5646.

(4) Shapiro, N. D.; Soh, S.; Mirica, K. A.; Whitesides, G. M. Anal. Chem. 2012, 84, 6166-6172.

(5) Mirica, K. A.; Ilievski, F.; Ellerbee, A. K.; Shevkoplyas, S. S.; Whitesides, G. M. Adv. Mater. 2011, 23, 4134-4140.

(6) Ilievski, F.; Mirica, K. A.; Ellerbee, A. K.; Whitesides, G. M. Soft Matter 2011, 7, 91139118.

(7) Winkleman, A.; Perez-Castillejos, R.; Gudiksen, K. L.; Phillips, S. T.; Prentiss, M.;

Whitesides, G. M. Anal. Chem. 2007, 79, 6542-6550.

(8) Lockett, M. R.; Mirica, K. A.; Mace, C. R.; Blackledge, R. D.; Whitesides, G. M. J. Forensic Sci. 2013, 58, 40-45. 
(9) Mirica, K. A.; Phillips, S. T.; Shevkoplyas, S. S.; Whitesides, G. M. J. Am. Chem. Soc. 2008, 130, 17678-17680.

(10) Yoshida, Y.; Saito, G. Z. J. Mater. Chem. 2006, 16, 1254-1262.

(11) Plechkova, N. V.; Seddon, K. R. Chem. Soc. Rev. 2008, 37, 123-150.

(12) Welton, T. Chem. Rev. 1999, 99, 2071-2083.

(13) Hallett, J. P.; Welton, T. Chem. Rev. 2011, 111, 3508-3576.

(14) Hayashi, S.; Hamaguchi, H. O. Chem. Lett. 2004, 33, 1590-1591.

(15) Del Sesto, R. E.; McCleskey, T. M.; Burrell, A. K.; Baker, G. A.; Thompson, J. D.; Scott, B. L.; Wilkes, J. S.; Williams, P. Chem. Commun. 2008, 447-449.

(16) Li, M.; De Rooy, S. L.; Bwambok, D. K.; El-Zahab, B.; DiTusa, J. F.; Warner, I. M. Chem. Commun. 2009, 6922-6924.

(17) Okuno, M.; Hamaguchi, H. O.; Hayashi, S. Appl. Phys. Lett. 2006, 89, 132506.

(18) Huddleston, J. G.; Willauer, H. D.; Swatloski, R. P.; Visser, A. E.; Rogers, R. D. Chem. Commun. 1998, 1765-1766.

(19) Visser, A. E.; Swatloski, R. P.; Griffin, S. T.; Hartman, D. H.; Rogers, R. D. Separ. Sci. Technol. 2001, 36, 785-804.

(20) Blanchard, L. A.; Hancu, D.; Beckman, E. J.; Brennecke, J. F. Nature 1999, 399, 28-29. (21) Jin, H.; O'Hare, B.; Dong, J.; Arzhantsev, S.; Baker, G. A.; Wishart, J. F.; Benesi, A. J.; Maroncelli, M. J. Phys. Chem. B 2008, 112, 81-92.

(22) Tokuda, H.; Tsuzuki, S.; Susan, M.; Hayamizu, K.; Watanabe, M. J. Phys. Chem. B 2006, 110, 19593-19600.

(23) Kolbeck, C.; Lehmann, J.; Lovelock, K. R. J.; Cremer, T.; Paape, N.; Wasserscheid, P.; Froba, A. P.; Maier, F.; Steinruck, H. P. J. Phys. Chem. B 2010, 114, 17025-17036.

(24) Geppert-Rybczynska, M.; Lehmann, J. K.; Peppel, T.; Kockerling, M.; Heintz, A. J. Chem. Eng. Data 2010, 55, 5534-5538. 
A)

$$
\begin{aligned}
& \mathrm{yR}-\mathrm{Cl}+\mathrm{M}-\mathrm{X}_{\mathrm{n}} \stackrel{\text { Neat or } \mathrm{MeOH}}{\stackrel{\text { r.t., } 12 \mathrm{~h}}{\longrightarrow}}[\mathrm{R}]_{\mathrm{y}}\left[\mathrm{MX}_{\mathrm{n}+\mathrm{y}}\right] \\
& \mathrm{R}=\mathrm{BMIM} \text {, Aliq, } \mathrm{AlaC}_{1} \\
& \mathrm{X}=\mathrm{Cl}, \mathrm{Br} \\
& \mathrm{M}=\mathrm{Fe}(\mathrm{y}=1, \mathrm{n}=3) ; \mathrm{Mn}(\mathrm{y}=2, \mathrm{n}=2) ; \mathrm{Gd}, \mathrm{Ho}, \mathrm{Dy}(\mathrm{y}=3, \mathrm{n}=3)
\end{aligned}
$$

B)

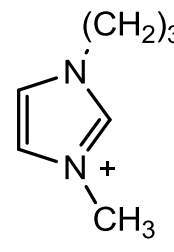

BMIM

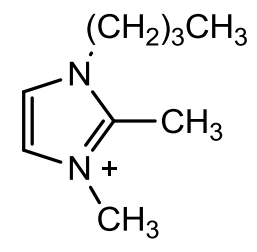

BDMIM

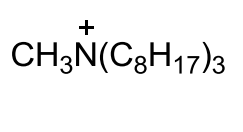

Aliq

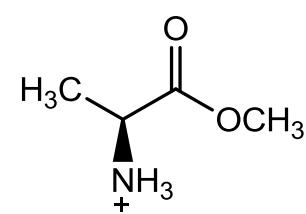

$\mathrm{AlaC}_{1}$

Scheme 1. a) Synthesis of PILs. b) Cations used in this study. [BMIM]: 1-butyl-3-methyl imidazolium; [BDMIM]: 1-butyl-2,3-dimethyl imidazolium; [Aliq]: Methyltrioctylammonium; $\left[\mathrm{AlaC}_{1}\right][\mathrm{Cl}]$ : Alanine methyl ester. 


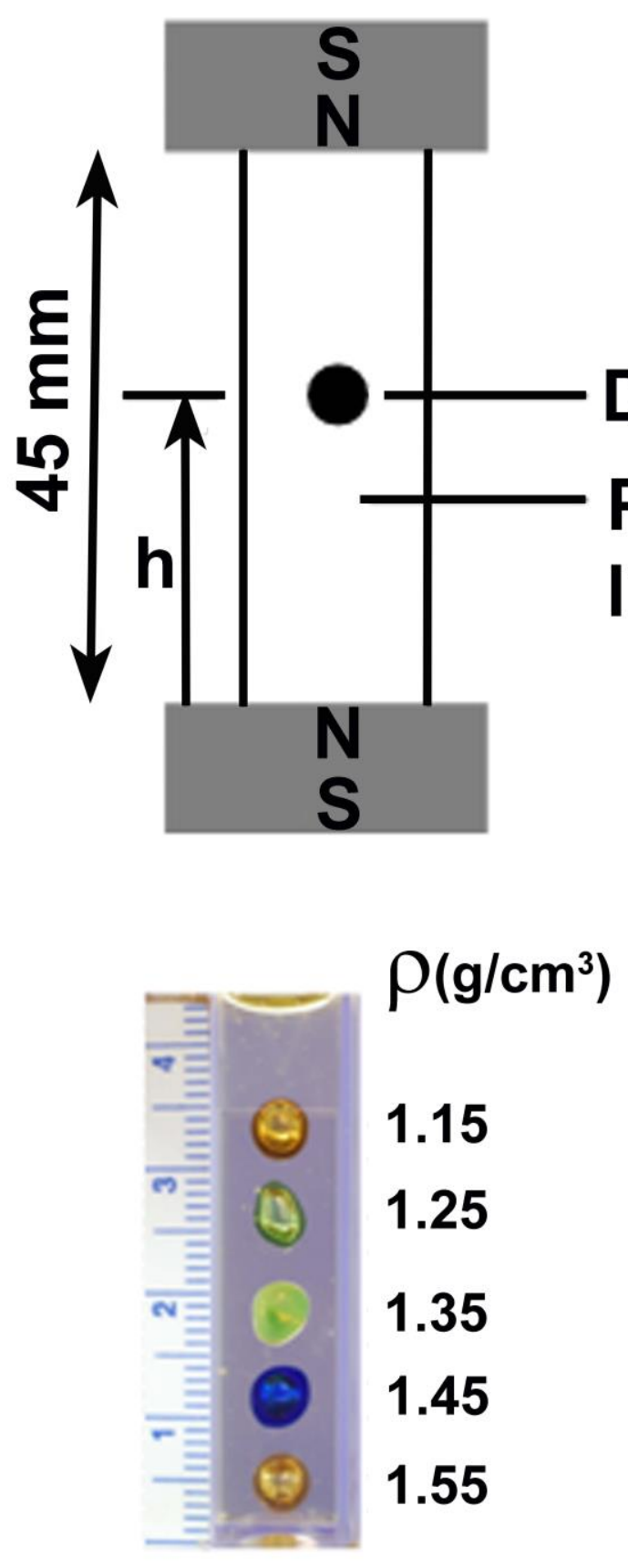

Figure 1. a) Schematic illustration of a typical MagLev device used for measuring the densities of diamagnetic objects using PILs as levitation medium. b) A picture showing density-standard beads levitated in MagLev using $[\mathrm{BDMIM}]_{3}\left[\mathrm{DyCl}_{6}\right]$ PIL. 

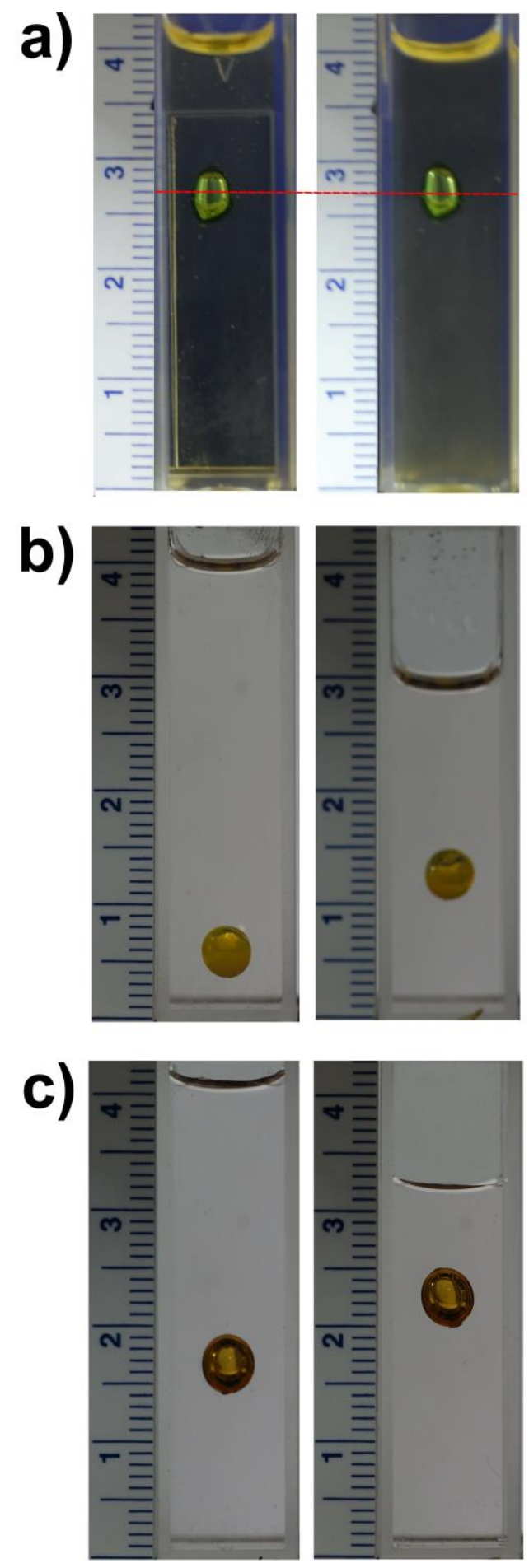

Figure 2. a) Photographs illustrating that a bead having known density levitate at the same height $\mathrm{h}(\mathrm{eq} 1)$ in non-volatile $[\mathrm{AlaC}]_{3}\left[\mathrm{DyCl}_{6}\right] \mathrm{PIL}$ (i) on the first day of the experiment and (ii) after one week (or heating to $100{ }^{\circ} \mathrm{C}$ and cooling). b) Photographs showing a density-standard 
bead levitate at different heights in an ethanolic solution of manganese chloride, (i) after 5 minutes in the MagLev device, and (ii) after 6 hours in the device, open to the atmosphere due to evaporation of solvent. c) Photographs showing that a density-standard bead levitates at different heights in an aqueous solution of manganese chloride, (i) after 5 minutes in the MagLev device, and (ii) after heating to $100^{\circ} \mathrm{C}$ for 5 minutes in an open vessel, and cooling. The evaporation of organic or aqueous solvents (at room temperature or by heating) increases the concentration and magnetic susceptibility of the paramagnetic medium. 

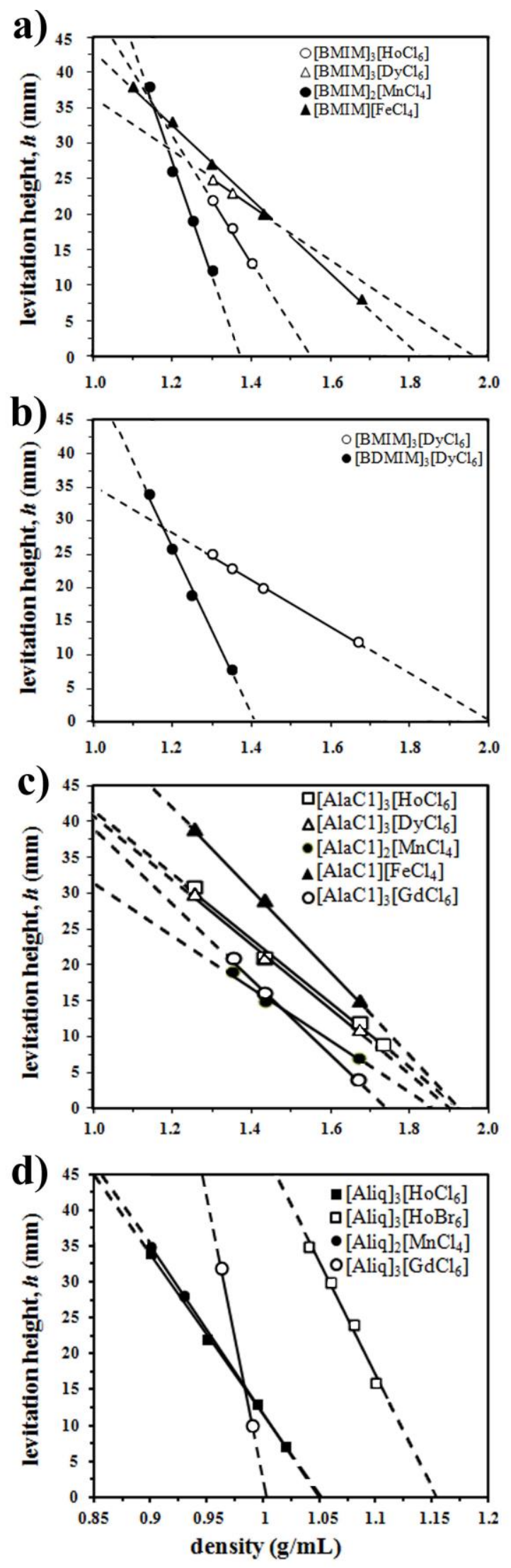
Figure 3. a) A graph showing the relationship between the levitation height and density of beads with known densities levitated in imidazolium-based PILs with different metal chloride anions. b) A comparison of PILs containing $\mathrm{DyCl}_{6}$ anion and two different imidazolium cations. c) A graph showing the relationship between the levitation height and density of beads with known densities levitated in L-alanine methyl ester cation but with different paramagnetic metal chloride anions. d) A graph showing the relationship between the levitation height and density of beads with known densities levitated in Aliquat ${ }^{\circledR}$-based PILs with different paramagnetic metal halide anions. 


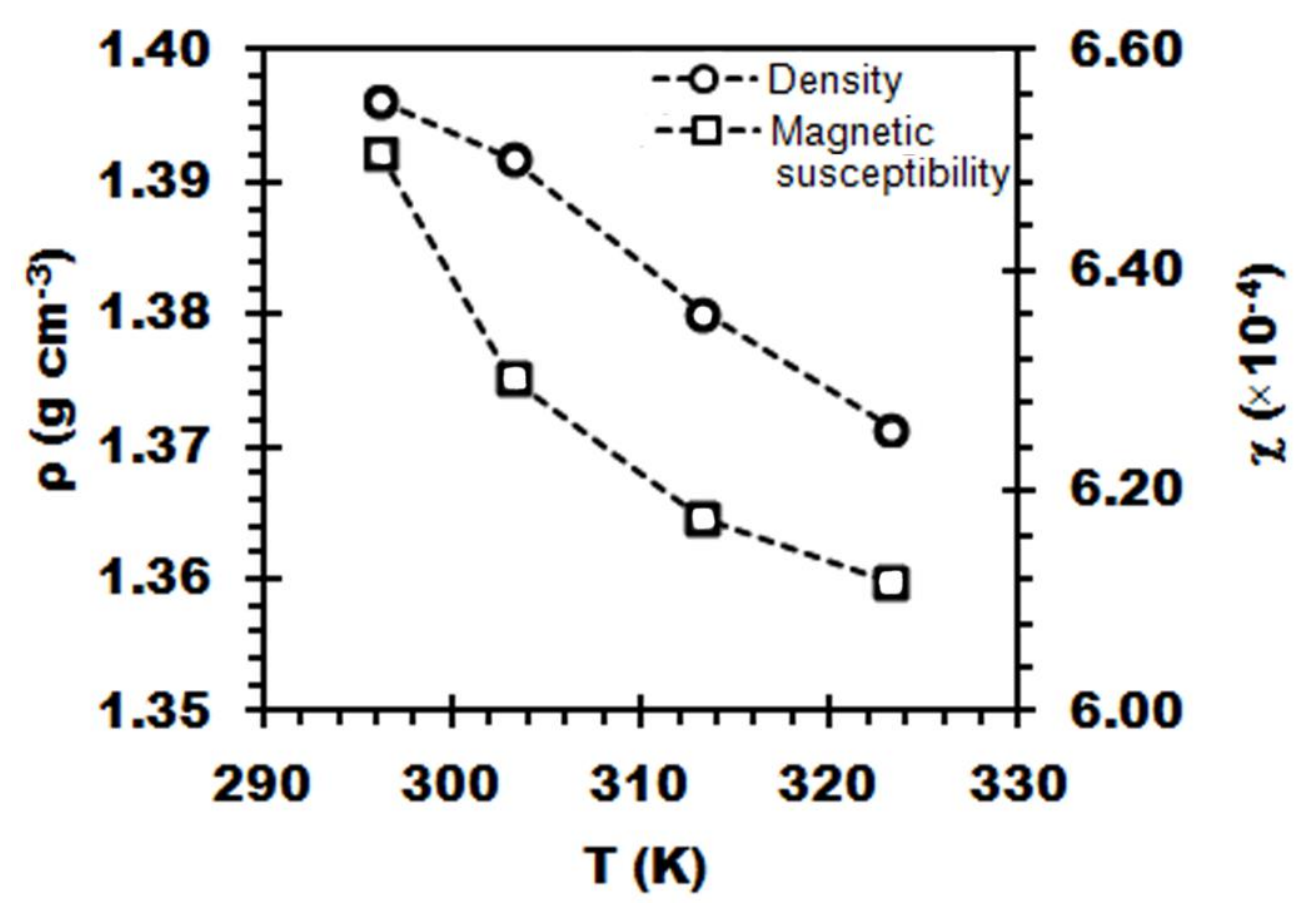

Figure 4. A graph showing the relationship between the density $(\rho)$ and magnetic susceptibility $(\chi)$ of $\left[\mathrm{AlaC}_{1}\right]_{3}\left[\mathrm{DyCl}_{6}\right]$ PIL, as a function of temperature $(\mathrm{T})$. 
a)
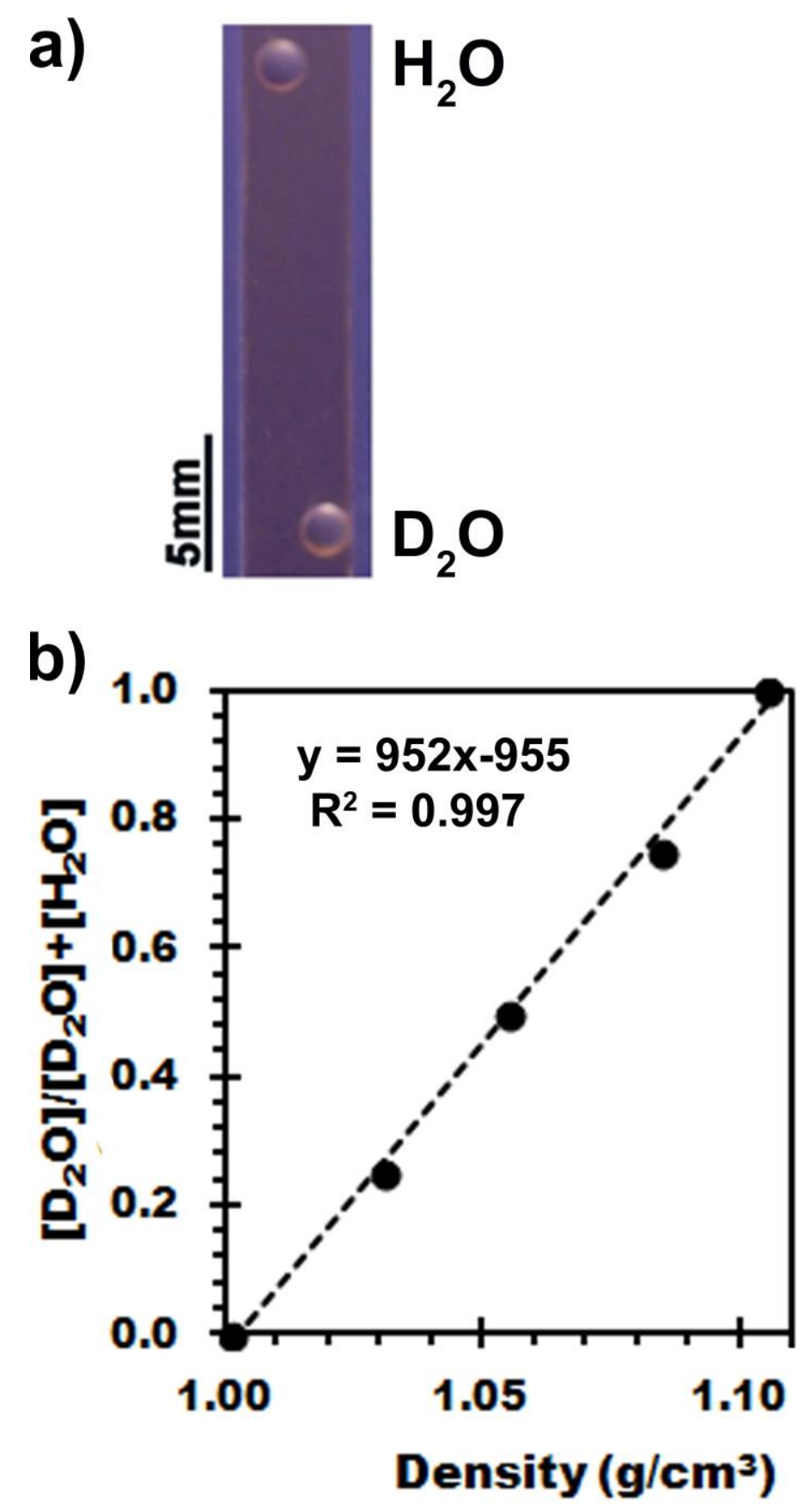

Figure 5. a) Picture showing droplets of water and deuterium oxide levitating in [Aliq $]_{3}\left[\mathrm{HoCl}_{6}\right]$ PIL. b) A graph showing the relationship between density and the isotopic ratio of $\mathrm{D}_{2} \mathrm{O}$ in the mixture with water. 
Table 1. (A) Physico-chemical, magnetic and thermal properties of PILs.

\begin{tabular}{|c|c|c|c|c|c|c|}
\hline PIL & $\begin{array}{c}\rho^{a} \\
\left(\mathrm{~g} / \mathrm{cm}^{3}\right)\end{array}$ & $\begin{array}{c}{\left[\mathbf{M}^{\mathbf{n}^{+}}\right]^{b}} \\
(\mathrm{~mol} / \mathbf{L})\end{array}$ & $\chi\left(\times 10^{-4}\right)$ & $\begin{array}{c}\text { Accessible } \\
\text { Density } \\
\text { Range } \\
\left(\mathrm{g} / \mathrm{cm}^{3}\right)\end{array}$ & $\mathbf{T}_{\mathrm{g}}\left({ }^{\circ} \mathbf{C}\right)^{c}$ & $\mathbf{T}_{\mathbf{d}}\left({ }^{\circ} \mathbf{C}\right)^{d}$ \\
\hline$[\mathrm{Aliq}]_{2}\left[\mathrm{MnCl}_{4}\right]$ & 0.96 & 1.03 & 1.85 & $0.90-1.00$ & $e$ & 248 \\
\hline$[\text { Aliq }]_{3}\left[\mathrm{GdCl}_{6}\right]$ & 0.97 & 0.66 & 0.55 & $0.95-0.99$ & $e$ & 181 \\
\hline$[\mathrm{Aliq}]_{3}\left[\mathrm{HoCl}_{6}\right]$ & 0.95 & 0.64 & 1.94 & $0.85-1.05$ & $e$ & 198 \\
\hline$[\text { Aliq }]_{3}\left[\mathrm{HoBr}_{6}\right]$ & 1.08 & 0.62 & 1.37 & $1.01-1.15$ & $e$ & 177 \\
\hline$[\mathrm{BMIM}]_{3}\left[\mathrm{HoCl}_{6}\right]$ & 1.29 & 1.62 & 5.40 & $1.30-1.35$ & $e$ & 298 \\
\hline$[\mathrm{BMIM}]\left[\mathrm{FeCl}_{4}\right]$ & 1.39 & 4.12 & 8.28 & $0.96-1.83$ & $-88^{\#}$ & 280 \\
\hline$[\mathrm{BDMIM}]_{2}\left[\mathrm{MnCl}_{4}\right]$ & 1.24 & 2.47 & 2.75 & $1.09-1.37$ & -44 & 333 \\
\hline$[\mathrm{BMIM}]_{3}\left[\mathrm{DyCl}_{6}\right]$ & 1.37 & 1.72 & 12.37 & $0.72-2.01$ & $e$ & 295 \\
\hline$[\mathrm{BDMIM}]_{3}\left[\mathrm{DyCl}_{6}\right]$ & 1.23 & 1.47 & 3.49 & $1.05-1.41$ & $e$ & 334 \\
\hline$[\mathrm{AlaC} 1]\left[\mathrm{FeCl}_{4}\right]$ & 1.52 & 5.04 & 6.62 & $1.15-1.93$ & -62 & 191 \\
\hline$[\mathrm{AlaC} 1]_{2}\left[\mathrm{MnCl}_{4}\right]$ & 1.24 & 3.06 & 11.80 & $0.63-1.86$ & -59 & 187 \\
\hline$[\mathrm{AlaC} 1]_{3}\left[\mathrm{GdCl}_{6}\right]$ & 1.31 & 1.92 & 8.24 & $0.89-1.74$ & -25 & 310 \\
\hline$[\mathrm{AlaC} 1]_{3}\left[\mathrm{HoCl}_{6}\right]$ & 1.42 & 2.06 & 9.70 & $0.92-1.93$ & -48 & 247 \\
\hline$[\mathrm{AlaC} 1]_{3}\left[\mathrm{DyCl}_{6}\right]$ & 1.41 & 2.05 & 9.58 & $0.91-1.91$ & -58 & 159 \\
\hline $4.5 \mathrm{M} \mathrm{MnCl}_{2(\mathrm{Aq})} *$ & 1.32 & 4.50 & 4.50 & $1.20-1.55$ & $n / a$ & $n / a$ \\
\hline
\end{tabular}

${ }^{a}$ Density $\left(\rho_{P L L}\right)$ and magnetic susceptibility $\left(\chi_{P L}\right)$ of each PIL were determined as previously described. ${ }^{1 b}\left[\mathrm{Mn}^{+}\right]$is the concentration of the paramagnetic ion in the PIL. ${ }^{c}$ Glass transition temperatures $\left(\mathrm{T}_{\mathrm{g}}\right)$ were measured using differential scanning calorimetry (DSC). ${ }^{d}$ Decomposition temperatures $\left(\mathrm{T}_{\mathrm{d}}\right)$ determined from thermal gravimetric analysis (TGA) were measured at the onset of decomposition ( $\sim 10 \%$ mass loss). ${ }^{e} \mathrm{~T}_{\mathrm{g}}$ not observed between $-75^{\circ} \mathrm{C}$ to $25^{\circ} \mathrm{C}$. ${ }^{*}$ Not a PIL. ${ }^{n / a}$ Not measured. ${ }^{\#}$ From literature. ${ }^{10}$ 
"For TOC only"

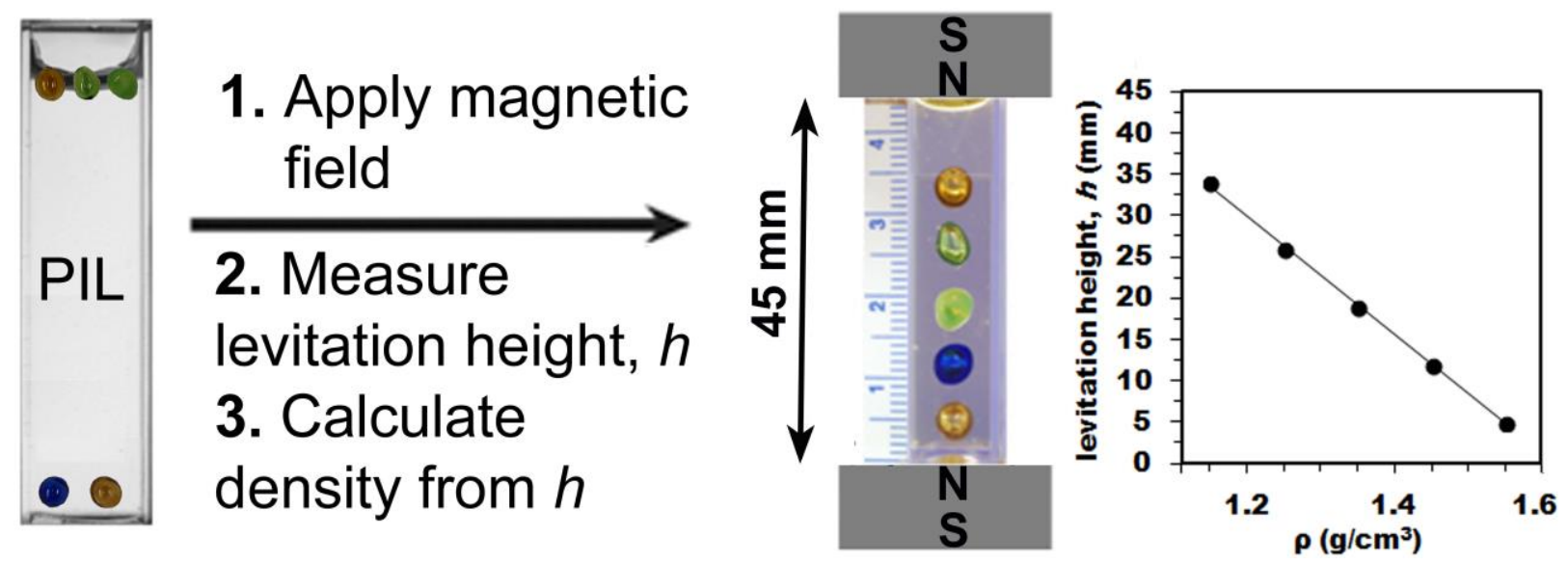

